\title{
Bacterial Transformation of Aromatic Monomers in Softwood Black Liquor
}

\author{
Laura E. Navas', Gara Dexter', Jie Liu', David Levy-Booth', MiJung Cho', \\ Soo-Kyeong Jang ${ }^{2}$, Shawn D. Mansfield ${ }^{2}$, Scott Renneckar ${ }^{2}$, William W. Mohn ${ }^{1}$ and \\ Lindsay D. Eltis ${ }^{1 *}$
}

${ }^{1}$ Department of Microbiology and Immunology, Life Sciences Institute, BioProducts Institute, The University of British Columbia, Vancouver, BC, Canada, ${ }^{2}$ Department of Wood Science, BioProducts Institute, The University of British Columbia, Vancouver, BC, Canada

The valorization of lignin, a major component of plant-derived biomass, is essential to sustainable biorefining. We identified the major monoaromatic compounds present in black liquor, a lignin-rich stream generated in the kraft pulping process, and investigated their bacterial transformation. Among tested solvents, acetone extracted the greatest amount of monoaromatic compounds from softwood black liquor, with guaiacol, vanillin, and acetovanillone, in an approximately 4:3:2 ratio, constituting 90\% of the total extracted monoaromatic content. 4-Ethanol guaiacol, vanillate, and 4-propanol guaiacol were also present. Bacterial strains that grew on minimal media supplemented with the BL extracts at $1 \mathrm{mM}$ total aromatic compounds included Pseudomonas putida KT2442, Sphingobium sp. SYK-6, and Rhodococcus rhodochrous EP4. By contrast, the extracts inhibited the growth of Rhodococcus jostii RHA1 and Rhodococcus opacus PD630, strains extensively studied for lignin valorization. Of the strains that grew on the extracts, only $R$. rhodochrous GD01 and GD02, isolated for their ability to grow on acetovanillone, depleted the major extracted monoaromatics. Genomic analyses revealed that EP4, GD01, and GD02 share an average nucleotide identity (ANI) of $98 \%$ and that GD01 and GD02 harbor a predicted three-component carboxylase not present in EP4. A representative carboxylase gene was upregulated 100-fold during growth of GD02 on a mixture of the BL monoaromatics, consistent with the involvement of the enzyme in acetovanillone catabolism. More generally, quantitative RT-PCR indicated that GD02 catabolizes the BL compounds in a convergent manner via the $\beta$-ketoadipate pathway. Overall, these studies help define the catabolic capabilities of potential biocatalytic strains, describe new isolates able to catabolize the major monoaromatic components of $\mathrm{BL}$, including acetovanillone, and facilitate the design of biocatalysts to valorize under-utilized components of industrial lignin streams.

Keywords: aromatic compound, bacterial catabolism, lignin, acetovanillone, Rhodococcus

\section{INTRODUCTION}

Lignin is a heterogeneous aromatic polymer and a major component of the plant cell wall. Although it can comprise over $30 \%$ of lignocellulosic biomass, it is under-utilized in biorefineries, typically being used to power the extraction of carbohydrates (Ragauskas et al., 2014; Becker and Wittmann, 2019). Technoeconomic analyses have identified the valorization of lignin as 
being critical for the economic viability and sustainability of next generation biorefineries. Accordingly, considerable effort has been invested in developing processes to depolymerize lignin and convert it to value-added bioproducts (Ragauskas et al., 2014; Schutyser et al., 2018; Sun et al., 2018; Becker and Wittmann, 2019). One under-utilized, lignin-rich stream that is generated in the kraft pulping process is black liquor (BL; Johnson et al., 2019). In an effort to valorize this fraction, processes such as LignoForce ${ }^{\mathrm{TM}}$ have been developed to recover the kraft lignin (Kouisni et al., 2016). BL also contains lower molecular weight compounds, including monoaromatics, sugars, and small organic acids that represent unmined value.

The ability of phylogenetically diverse bacteria to efficiently catabolize aromatic compounds has provided an entry to developing microbial cell factories to upgrade lignin-derived aromatic compounds (LDACs) to commodity chemicals (Becker and Wittmann, 2019). Strains that have been investigated for their biocatalytic potential include Sphingobium sp. SYK-6 (Kamimura et al., 2017), Pseudomonas putida KT2440 (Weimer et al., 2020), Novosphingobium aromaticivorans DSM 12444 (Perez et al., 2021), Rhodococcus opacus PD630 (Kosa and Ragauskas, 2012), and Rhodococcus jostii RHA1 (Eltis and Singh, 2018). More recently, we isolated Rhodococcus rhodochrous EP4 for its ability to grow on alkylated guaiacols generated in the reductive catalytic fractionation (RCF) of hardwood lignin (Levy-Booth et al., 2019; Fetherolf et al., 2020). These strains not only have exceptional abilities to aerobically degrade LDACs, but also have a high natural resistance to toxic compounds (Krell et al., 2012; Xu et al., 2019). Bacteria of the genus Rhodococcus are of particular interest for industrial applications given their use to produce thousands of tons of acrylamide (Hughes et al., 1998).

In bacterial strains, the aerobic catabolism of aromatic compounds is normally organized in a convergent fashion, whereby "upper pathways" transform a diversity of substrates to a small number of shared intermediates, typically catechols, which are further transformed to central metabolites through "lower pathways" (Eltis and Singh, 2018; Becker and Wittmann, 2019). For example, in RHA1, upper pathways transform $p$-coumarate, ferulate, vanillate, and 4-hydroxybenzoate to protocatechuate, which is then transformed to acetyl-CoA and succinate via the $\beta$-ketoadipate lower pathway (Patrauchan et al., 2005; Chen et al., 2012; Otani et al., 2014). This convergent catabolism facilitates the engineering of microbial cell factories to "biologically funnel" mixtures of LDACs to commercially valuable compounds in high atom yield. For example, KT2440 has been engineered to transform alkaline pretreated lignin into muconic acid, which can in turn be hydrogenated to adipic acid, a precursor for nylon (Vardon et al., 2015). More recently, this strain was engineered to convert vanillin and vanillate from softwood BL to $\beta$-ketoadipate (Suzuki et al., 2021). Such compounds with rich functionality are more readily accessible from biomass than petroleum and can be used to develop platform chemicals and novel materials (Johnson et al., 2019).

A number of studies have investigated the potential of bacteria to transform softwood kraft lignin. In one study, Abdelaziz et al. (2019) oxidatively depolymerized lignin to a mixture of vanillin, vanillate, guaiacol, and acetovanillone. They further demonstrated that SYK-6, KT2440, and a rhodococcal strain grew on this stream. However, none of these strains completely degraded all the LDACs. Similarly, Ravi et al. (2019) used an alkaline depolymerized softwood kraft lignin as substrate for bacterial conversion, demonstrating that two Pseudomonas strains grew on vanillate, vanillin, and 4-hydroxybenzoate, while a rhodococcal strain degraded these compounds as well as guaiacol. In this study, acetovanillone remained as the only persistent monoaromatic. Finally, Paenibacillus glucanolyticus SLM1, was isolated from BL, grew on this substrate, and degraded some of the lignin to monoaromatic compounds (Mathews et al., 2014, 2016). However, the consumption of the LDACs was not analyzed.

Herein, we characterized the monoaromatic extract of softwood BL and the relative efficiency of different extraction methods. We then evaluated the ability of seven bacterial strains to grow on the $\mathrm{BL}$ extract and its major monoaromatic components. These included two $R$. rhodochrous strains, GD01 and GD02 that we isolated on acetovanillone and whose genomes we sequenced. Finally, we characterized growth of GD02 on the BL monoaromatic components and evaluated the involvement of predicted catabolic pathways in this growth. The results provide insights into the bacterial catabolism of LDACs present in an industrially relevant liquor stream and facilitate the design of biocatalysts for lignin valorization.

\section{MATERIALS AND METHODS}

\section{Chemicals and Reagents}

All reagents were of analytical grade unless otherwise noted. 4-Propanol guaiacol (4PG) was synthesized and generously provided by Dr. Rui Katahira according to the method from Pepper et al. (1971).

\section{Characterization of BL Extracts}

Black liquor was produced by Domtar Corporation (Canada) using the Lignoforce ${ }^{\mathrm{TM}} \mathrm{kraft}$ pulping process and a mixture of white wood that included spruce, pine, and fir (Kouisni et al., 2016). Compounds were extracted from $20 \mathrm{ml}$ BL using $5 \mathrm{ml}$ of either ethyl acetate, diethyl ether, dichloromethane, or acetone. Extraction mixtures were agitated by shaker for $2 \mathrm{~h}$ $(200 \mathrm{rpm})$, and were stabilized for $30 \mathrm{~min}$ prior to phase separation.

\section{GC-MS Analysis}

Aromatic compounds in extracts were analyzed using an Agilent Technologies (Santa Clara, U.S.A.) 6890 N gas chromatograph equipped with a 30-m Agilent 190915-433 capillary column and an Agilent 5973 mass-selective detector. For monoaromatic quantification, samples were dried and derivatized using $\mathrm{N}, \mathrm{O}$ bis(trimethylsilyl)trifluoroacetamide and trimethylchlorosilane in a 50/50 mixture with pyridine. Runs were held at $90^{\circ} \mathrm{C}$ for $3 \mathrm{~min}$, and then ramped to $290^{\circ} \mathrm{C}$ at $12^{\circ} \mathrm{C} \mathrm{min}^{-1}$ with a $10 \mathrm{~min}$ final hold. Standard curves of each compound were 
run in parallel. To evaluate the substrate depletion in culture supernatant, $400 \mu \mathrm{l}$ samples were withdrawn, spiked with $5 \mathrm{nmol}$ of 3-chlorobenzoate as an internal standard, and extracted with an equal volume of ethyl acetate. The extracted compounds were dried under a stream of $\mathrm{N}_{2}$ and processed as described above. Samples were run in triplicate.

\section{HPLC Analysis}

Organic acids in extracts were quantified using a Summit highperformance liquid chromatography (HPLC) apparatus equipped with a Shodex RI-101 detector (Dionex, United States) and an ICSep ION-300 column $(300 \mathrm{~mm} \times 7.8 \mathrm{~mm}$, Transgenomic, United States). The mobile phase was $0.0085 \mathrm{~N} \mathrm{H}_{2} \mathrm{SO}_{4}$. The column was operated at a flow rate of $0.5 \mathrm{ml} \mathrm{min}^{-1}$ at $65^{\circ} \mathrm{C}$. Samples of $20 \mu \mathrm{l}$ were injected and compounds were quantified using standard curves.

For experiments on the transformation of $4 \mathrm{PG}$ and 4-ethanol guaiacol (4EG) by GD02, culture supernatants were analyzed using a Waters 2695 HPLC (Waters, Milford, MA, United States) equipped with a $250 \times 4.6 \mathrm{~mm}$ Luna $^{\circledR} 5 \mu \mathrm{m} \mathrm{C18}(2)$ column (Phenomenex, Torrance, CA, United States) and a UV detector. The column was operated at $0.7 \mathrm{mlmin}^{-1}$, and the sample was eluted using a $16.8 \mathrm{ml}$ linear gradient of $0.1 \%$ formic acid in $\mathrm{H}_{2} \mathrm{O}$ to $100 \%$ methanol. Filtered $(0.2 \mu \mathrm{m})$ samples of $100 \mu \mathrm{l}$ were injected.

\section{Bacterial Strains and Growth Conditions}

The bacterial strains used in this study were P. putida KT2442, Sphingobium sp. SYK-6, R. opacus PD630, and $R$. jostii RHA1, and three strains of $R$. rhodochrous: EP4, GD01, and GD02. EP4 was recently isolated by Levy-Booth et al. (2019), and GD01 and GD02 were isolated during this study (see below). Strains were grown at $30^{\circ} \mathrm{C}$ on LB or $\mathrm{M} 9$ minimal medium supplemented with Goodies (Bauchop and Elsden, 1960) and defined organic substrates. Growth experiments were routinely performed in 250-ml shake flasks containing $50 \mathrm{ml}$ of culture and monitoring the optical density at $600 \mathrm{~nm}\left(\mathrm{OD}_{600}\right)$. Growth studies in 96-well plate were performed using a Tecan Spark-Multimode Microplate Reader with shaking at $250 \mathrm{rpm}$ and $\mathrm{OD}_{600}$ recorded every $30 \mathrm{~min}$. Experiments were performed in triplicate.

For growth on individual substrates, substrate mixtures, and extracts, single colonies were inoculated in $5 \mathrm{ml} \mathrm{LB}$ broth and grown overnight. Cells were pelleted at $1,500 \times g$, washed twice with M9 media, and then used to inoculate M9-Goodies containing the substrate at an $\mathrm{OD}_{600} \sim 0.05$. For growth on acetone $\mathrm{BL}$ extracts, solvent was evaporated under $\mathrm{N}_{2}$ and suspended in DMSO to prepare a $100 \times$ stock solution $(100 \mathrm{mM})$. Due to the turbidity of the extract, growth was evaluated by counting CFU.

For inhibition experiments, LB broth was amended with BL extract to a total concentration of $1 \mathrm{mM}$ monoaromatic compounds and inoculated to an $\mathrm{OD}_{600} \sim 0.05$ with cells grown overnight in the same media. LB without BL extract served as a control.

\section{Enrichment and Isolation}

Enrichment cultures were inoculated with compost from The University of British Columbia farm, contained $1 \mathrm{mM}$ acetovanillone
( $\geq 98 \%$ Sigma-Aldrich, St. Louis. United States) as sole carbon source in M9 with Goodies, and were incubated with shaking at $200 \mathrm{rpm}$ at either 30 or $37^{\circ} \mathrm{C}$. The cultures were serially transferred three times, with acetovanillone increased to $2 \mathrm{mM}$ for the final two passages. Isolates were then obtained by streaking cultures on homologous media with $1 \mathrm{mM}$ acetovanillone and $1.5 \%$ agar. Individual colonies were streaked three times successively to obtain pure isolates. Isolates were tested for growth on $1 \mathrm{mM}$ acetovanillone in liquid medium, and the removal of acetovanillone was measured by gas chromatography-mass spectrometry (GC-MS). Only two of nine isolates grew on acetovanillone, GD01, isolated at $37^{\circ} \mathrm{C}$, and GD02, isolated at $30^{\circ} \mathrm{C}$.

\section{Transformation of 4PG and 4EG by GD02 Cells}

Cells of GD02 were grown overnight in $5 \mathrm{ml} \mathrm{LB}$ and washed as described in "Bacterial Strains and Growth Conditions." These cells were used to inoculate M9-Goodies supplemented with $1 \mathrm{mM}$ mixture of monoaromatics in the same proportion as in the $\mathrm{BL}$ acetone extract. The medium was inoculated to an $\mathrm{OD}_{600} \sim 0.05$ and cells were harvested when the culture reached mid-log phase. The harvested cells were concentrated $25 \times$ and incubated with each of $10 \mathrm{mM} 4 \mathrm{PG}$ and $4 \mathrm{EG}$ at $30^{\circ} \mathrm{C}$. Supernatant samples were withdrawn at different times, acidified with acetic acid, and analyzed using HPLC.

\section{Genome Sequencing and Bioinformatics}

Genomic DNA was extracted from strains GD01 and GD02 using FastDNA SPIN kit for soil (MPBio, Solon, OH, United States). Illumina sequencing was performed by the Microbial Genome Sequencing Center (MiGS, Pittsburgh, PA, United States) on the NextSeq2000 platform, and generated 3.3 million 150-bp pair-end reads with 111-fold sequencing coverage. De novo draft genomes were assembled by first processing and decontaminating reads with BBMap (Bushnell et al., 2017). Quality filtering and trimming of reads used BBDuk (Bushnell et al., 2017). Cleaned and filtered reads were then assembled using SPAdes 3.13.0 (Bankevich et al., 2012), MeDuSa 1.6 scaffolding (Bosi et al., 2015), and gap filling used SSPACE (Boetzer et al., 2011). Gene annotation used DIAMOND (Buchfink et al., 2015) and BLASTp against the Protein Data Bank (threshold of $E$-value $10^{-3}$ ). Average nucleotide identity (ANI) was calculated using FastANI (Jain et al., 2018).

\section{RNA Extraction and RT-qPCR}

Cultures for quantitative reverse transcription PCR (RT-qPCR) were grown on M9-Goodies supplemented with $1 \mathrm{mM}$ mixture of monoaromatics in the same proportion as in the BL acetone extract or $1 \mathrm{mM}$ citrate and harvested after $7 \mathrm{~h}\left(\mathrm{OD}_{600} 0.13\right)$ and $11 \mathrm{~h}\left(\mathrm{OD}_{600} 0.25\right)$. Cells were flash frozen and stored at $-80^{\circ} \mathrm{C}$ for later RNA extractions. Total RNA was extracted using Trizol and cleaned up by PureLink RNA mini kit (Thermo Fisher Scientific). Cells were suspended in Trizol and disrupted using a bead beater (MP Biomedicals FastPrep-24 Solon, OH) with six rounds of $30 \mathrm{~s}$ with speed set at $5.5 \mathrm{~m} \mathrm{~s}^{-1}$, and $5 \mathrm{~min}$ pauses between rounds. RNA samples were further treated 
with Turbo DNase I (Thermo Fisher Scientific) and the DNase Inactivation Reagent. RNA was reverse transcribed to cDNA using SuperScript ${ }^{\mathrm{TM}}$ VILO $^{\mathrm{TM}}$ cDNA Synthesis Kit (Thermo Fisher Scientific). Quantitative PCR was performed using a StepOne Plus Real-Time PCR System (Thermo Fisher Scientific) using the PrimeTime Gene Expression master mix (Integrated DNA Technologies). Primers and probes are listed in Supplementary Table 1. The cycling conditions were: $95^{\circ} \mathrm{C}$ for $3 \mathrm{~min}$, then $40 \mathrm{cycles}$ of: $95^{\circ} \mathrm{C}$ for $5 \mathrm{~s}$ and $60^{\circ} \mathrm{C}$ for $30 \mathrm{~s}$. Standard curves for all genes were made using $10 \times$ serial dilutions of synthesized target DNA gblocks from ( $0.1 \mathrm{ng}-0.1 \mathrm{fg})$. Transcript levels were normalized to the GD02 sigA cDNA and compared to expression in GD02 cells growing on $1 \mathrm{mM}$ citrate.

\section{RESULTS}

\section{Identification of Monoaromatic Compounds in BL}

To identify the aromatic compounds present in BL and to evaluate the efficiency of their isolation, we extracted softwood $\mathrm{BL}$ that had been subjected to the Lignoforce ${ }^{\mathrm{TM}}$ process with each of four solvents: ethyl acetate, diethyl ether, dichloromethane, and acetone. As described in Supplementary Material, the Lignoforce $^{\mathrm{TM}}$ process involves first oxidizing the $\mathrm{BL}$, then neutralizing it with $\mathrm{CO}_{2}$ and then rinsing with sulfuric acid to yield the $\mathrm{BL}$ filtrate for liquid extraction (Kouisni et al., 2016). Using a single extraction with $1: 4(\mathrm{v} / \mathrm{v})$ solvent:BL, the greatest total amount of monoaromatic compounds was obtained using acetone while diethyl ether yielded the least (Table 1).
In the acetone extract, guaiacol, vanillin, and acetovanillone were the most abundant monoaromatic compounds, collectively constituting $\sim 90 \%$ of the total monoaromatic content in an approximately 4:3:2 molar ratio. 4EG, 4PG, catechol, and vanillate were also detected. Comparison with the $\mathrm{BL}$ indicated that acetone extracted $35 \%$ of the neutral aromatic compounds from the $\mathrm{BL}$ and $3 \%$ of the vanillic acid. The acetone extract also contained significant quantities of formate and acetate (Table 1). By comparison, diethyl ether extracted around half the neutral aromatic compounds that acetone did.

\section{Isolation of $\boldsymbol{R}$. rhodochrous GD01 and GD02}

The identification of acetovanillone in $\mathrm{BL}$ extracts prompted us to isolate strains able to grow on this compound. Enrichment cultures were inoculated with compost and had acetovanillone as the sole organic substrate. After serial transfers of the enrichment cultures and streaking on homologous solid medium, two isolates that grew on acetovanillone were obtained and identified as GD01 and GD02. Sequencing the genomes of the strains yielded assemblies of 6.35 and $6.29 \mathrm{Mb}$ for GD01 and GD02, respectively, that were estimated to be $99.2 \%$ complete (Supplementary Table 2). GD01 and GD02 were classified as $R$. rhodochrous strains (Figure 1) based on the nucleotide sequence of their $16 \mathrm{~S}$ rRNA genes, ANI, and phylogenetic placement (Chaumeil et al., 2019). The 16S rRNA genes (27F1492R; Lane, 1991) of GD01 and GD02 shared 100\% sequence identity with that of $R$. rhodochrous EP4 (Levy-Booth et al., 2019). More generally, the GD01 and GD02 genomes were very closely related to each other, with an ANI of $99.1 \%$, as well as to $5.72 \mathrm{Mb}$ genome of EP4 (ANI 97.9\%).

TABLE 1 | Components of Lignoforce ${ }^{\mathrm{TM}}$ industrial black liquor (BL) filtrate from mixed softwood species ${ }^{\mathrm{a}}$.

\begin{tabular}{|c|c|c|c|c|}
\hline \multirow{2}{*}{ Parameter/component } & \multicolumn{4}{|c|}{ Extraction solvent } \\
\hline & Acetone & Dichloromethane & Diethyl ether & Ethyl acetate \\
\hline$\delta \mathrm{D}, \delta \mathrm{P}, \delta \mathrm{H}^{\mathrm{b}}$ & $15.5,10.4,7$ & $17,7.3,7.1$ & $14.5,2.9,4.6$ & $15.8,5.3,7.2$ \\
\hline Total solids (wt\%) & 2.8 & 0.5 & 0.5 & 0.5 \\
\hline Guaiacolc $^{c}$ & 7.4 & 8.8 & 6.6 & 8.0 \\
\hline Vanillin & 5.5 & 2.9 & 1.1 & 4.3 \\
\hline Acetovanillone & 3.5 & 3.4 & 1.5 & 3.6 \\
\hline 4-propanol guaiacol & 0.6 & 0.4 & 0.3 & 0.5 \\
\hline 4-ethanol guaiacol & 0.4 & 0.2 & 0.1 & 0.3 \\
\hline Catechol & 0.9 & $N^{d}$ & 0.3 & 0.6 \\
\hline Vanillic acid & 0.5 & ND & ND & ND \\
\hline Total phenolics (mM) ${ }^{\mathrm{e}}$ & 18.8 & 15.7 & 9.9 & 17.3 \\
\hline Extraction efficiency $(\%)^{\dagger}$ & 34.9 & 29.9 & 18.9 & 33.0 \\
\hline Formic acid & 101 & 17 & 22 & 34 \\
\hline Acetic acid & 38 & ND & ND & ND \\
\hline Succinic acid & 0.8 & ND & ND & ND \\
\hline Total acids $(\mathrm{mM})^{e}$ & 140 & 17 & 22 & 34 \\
\hline
\end{tabular}

aspruce, pine, and fir.

${ }^{b}$ Hansen solubility parameters: dispersion, polar, and hydrogen bonding components of cohesive energy density of the solvent (Hansen, 2007).

${ }^{\circ}$ Concentration of compounds in extracts provided in $\mathrm{mM}$ based on triplicate extractions. Errors did not exceed $5 \%$.

${ }^{d} \mathrm{ND}$, not detected.

eTrace amounts ( 0.1 mM) of lactic acid, 2-hydroxybutyric acid, 4-hydroxy-2-pentenoic acid, 4-hydroxy-3-pentenoic acid, and 2,5-dimethoxybenzoic acid were also detected in the acetone extract, but not in any of the others.

${ }^{\mathrm{f}}$ Estimated for neutral monoaromatics. 
GD02 grew on $2 \mathrm{mM}$ acetovanillone to stationary phase within $100 \mathrm{~h}$ in shake flasks (Figure 2A). Growth on acetovanillone was verified by plating CFUs (Figure 2B). GC-MS analysis indicated that acetovanillone was completely removed from the medium during growth (Figure 2A), with no metabolites detected. EP4 did not grow on acetovanillone.

\section{Five Bacterial Strains Grew on BL Extracts}

We assessed the toxicity of the BL extracts against a panel of seven bacterial strains known to grow on one or more of the major aromatic components of the BL: Sphingobium sp. SYK-6 (Kamimura et al., 2017), P. putida KT2442 (Weimer et al., 2020), R. jostii RHA1 (Eltis and Singh, 2018), R. opacus PD630
(Kosa and Ragauskas, 2012), R. rhodochrous EP4 (Fetherolf et al., 2020), $R$. rhodochrous GD01, and $R$. rhodochrous GD02. These strains were phylogenetically diverse, with the exception of the EP4, GD01, and GD02, which were included due to their distinct growth phenotypes.

For the toxicity experiments, solvents were evaporated from the different extracts and the residual materials were suspended in DMSO. Extracts were added to LB media such that the final concentration of $\mathrm{BL}$ aromatic compounds was $1 \mathrm{mM}$ (final 1\% DMSO). Among the seven tested strains, RHA1 and PD630 were the only ones to be completely inhibited by all four extracts (Supplementary Figure 1). At the other end of the spectrum, KT2442 and SYK-6 were the only strains whose

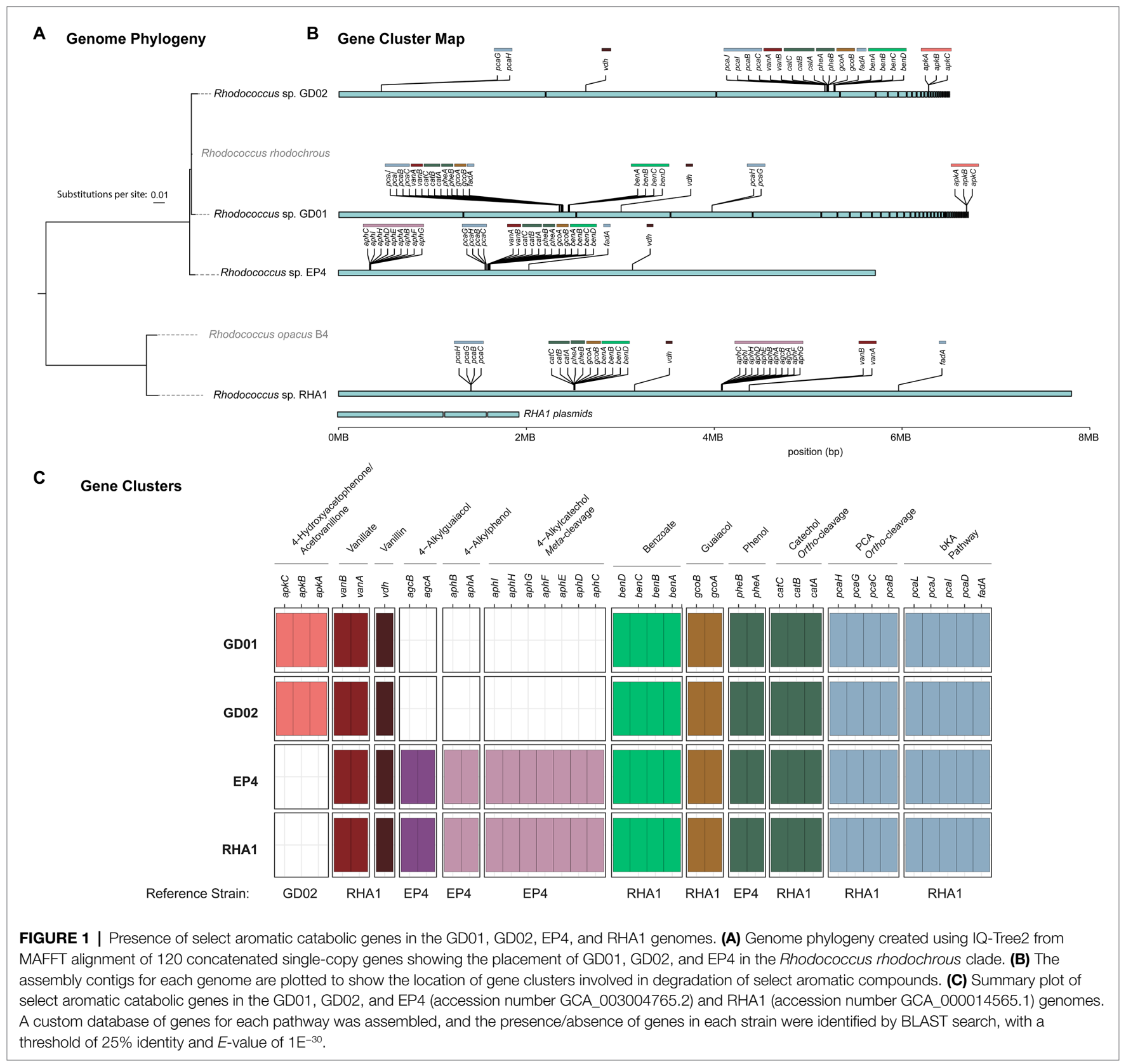



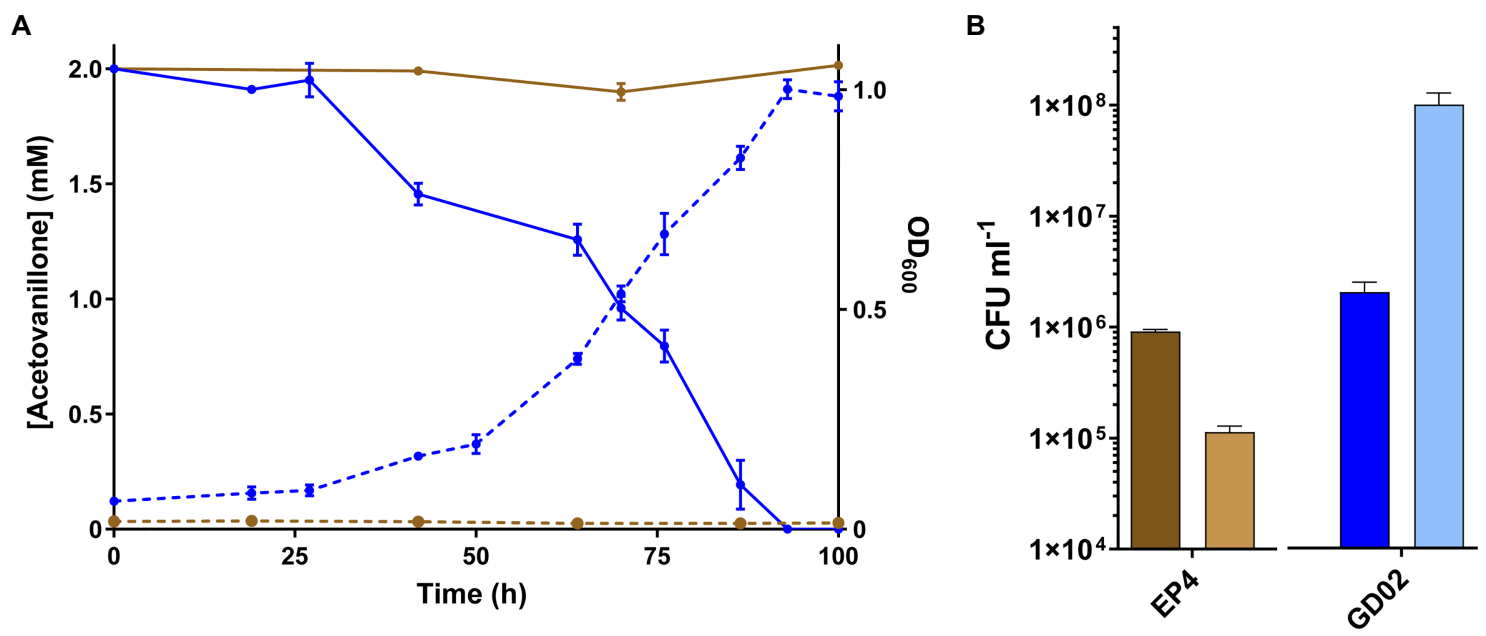

FIGURE 2 | Growth of GD02 on acetovanillone. (A) GD02 was grown in M9 minimal medium with 2 mM acetovanillone at $30^{\circ} \mathrm{C}$. Solid lines represent concentration of acetovanillone as determined using GC-MS, and dashed lines represent $\mathrm{OD}_{600}$. Blue and brown lines represent data for GD02 and EP4, respectively. (B) CFU ml-1 at $\mathrm{Oh}$ (dark) and $100 \mathrm{~h}$ (light). Data points show average of triplicate experiments and the vertical bars the SD.

growth on LB was not inhibited by any of the four extracts. The growth of EP4, GD01, and GD02 was delayed by the addition of acetone extract (i.e., longer lag phase), and was inhibited by the other three extracts. Nevertheless, the addition of the acetone extract increased the growth yield of the three $R$. rhodochrous strains, consistent with assimilation of BL-derived compounds. The dichloromethane and diethyl ether extracts were the most toxic, completely inhibiting the growth of the five Rhodococcus strains. Overall, these results indicate that the acetone-extracted $\mathrm{BL}$ is the least toxic of the extracts. Consequently, it was selected for further experiments as a growth substrate.

To study strains able to catabolize monoaromatics present in BL, we first assessed the growth of KT2442, SYK-6, EP4, GD01, and GD02 on BL acetone extracts such that the final concentration of monoaromatics was $1 \mathrm{mM}$. All strains showed robust growth, undergoing at least five doublings (Figure 3). Although GD01 underwent the greatest number of doublings, it was the only strain that did not reach stationary phase within $72 \mathrm{~h}$. A similar experiment was performed in a plate reader using a mixture of the aromatic compounds in the same proportion as in the $\mathrm{BL}$ acetone extract to a total concentration of $2 \mathrm{mM}$ as sole growth substrate in minimal medium (Supplementary Figure 2). Consistent with the growth on BL extracts, GD01 and GD02 grew to the highest OD on this mixture. The relatively low yield of KT2442 on the compound mixture vs. on the acetone extract could be due to the presence of additional substrates in the extracts, such as formate and acetate (Table 1).

\section{Depletion of Monoaromatic Compounds From BL Extracts}

To identify the aromatic compounds in the BL extract depleted by each strain, we analyzed spent culture supernatants using GC-MS. Catechol was not detected in the no-strain control,

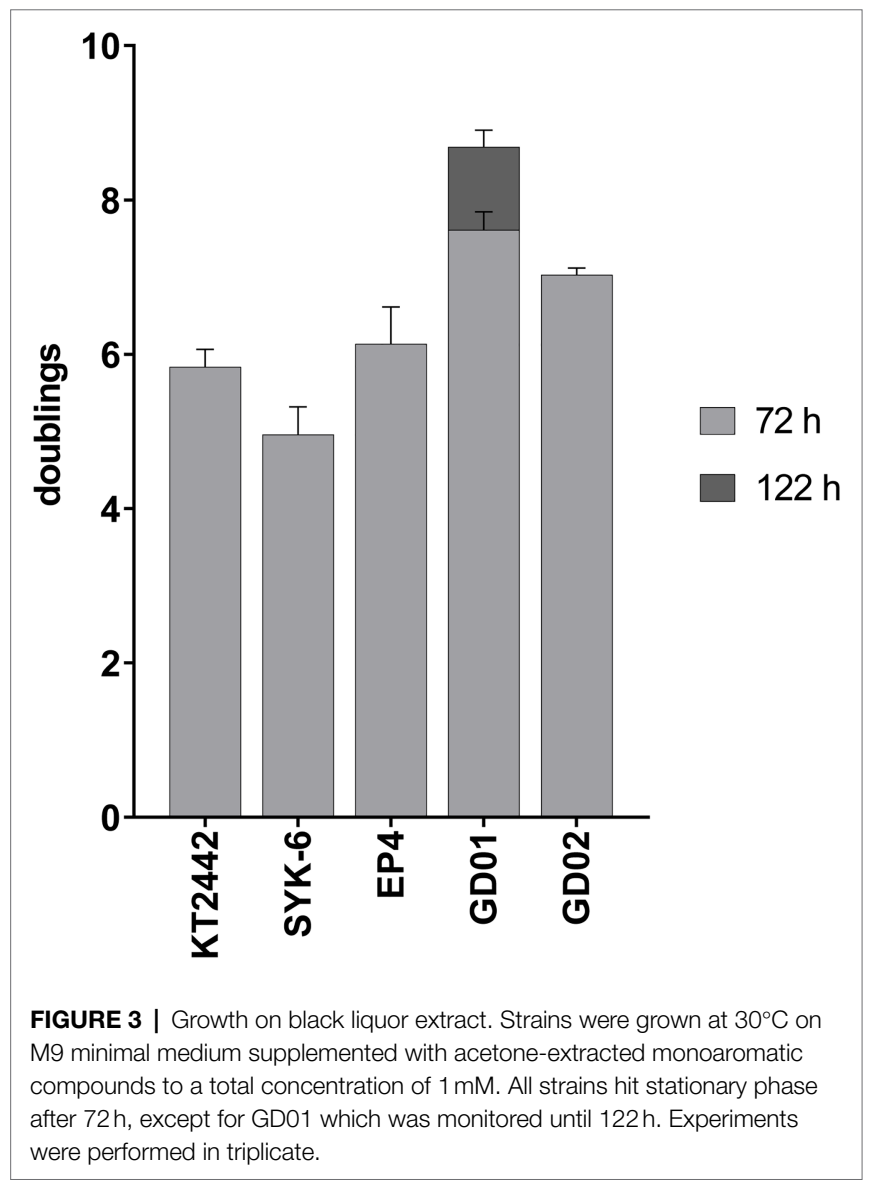

presumably due to its non-enzymatic oxidation. As summarized in Figure 4, only GD01 and GD02 depleted the six major monoaromatic compounds in the acetone extract after $72 \mathrm{~h}$. EP4 depleted all the compounds except acetovanillone. Among 


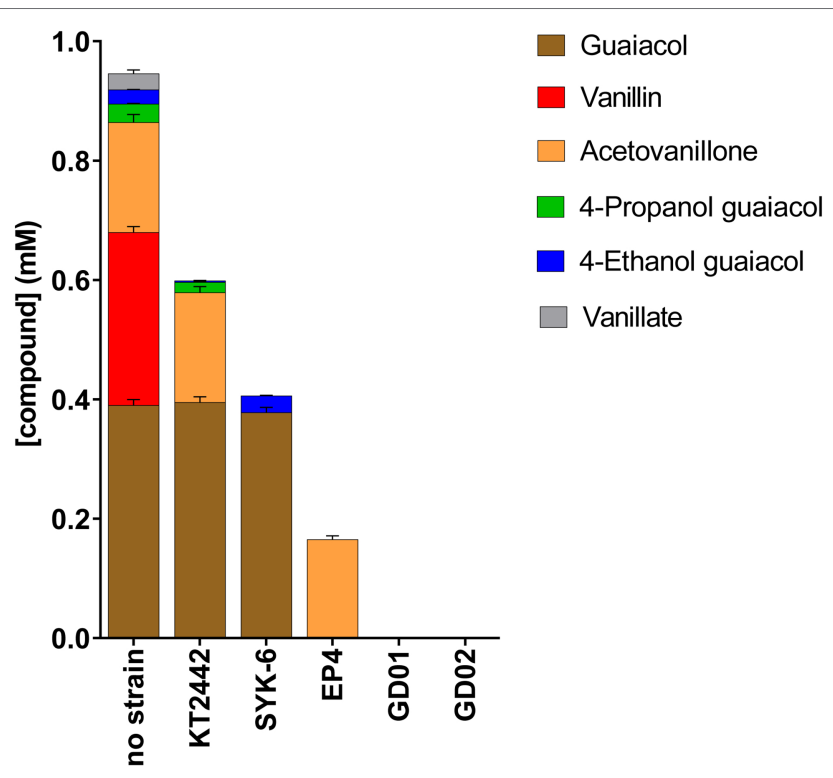

FIGURE 4 | Depletion of black liquor aromatic compounds by bacterial strains. Strains were incubated for $72 \mathrm{~h}$ on minimal medium amended with $\mathrm{BL}$ extract for a final concentration of $1 \mathrm{mM}$ total monoaromatic compounds. Compounds were quantified using GC-MS.

the non-rhodococcal strains, KT2442 completely depleted the vanillin and vanillate, and some of the 4EG. SYK-6 did not detectably deplete the guaiacol or 4EG, but completely depleted the other monomers, including acetovanillone. However, the SYK-6 did not grow on acetovanillone alone. Moreover, in monitoring the depletion of acetovanillone in the presence of each of the other monoaromatics, we found that this depletion occurred in the presence of vanillate. Consistent with vanillin being catabolized via vanillate, the addition of vanillin also induced acetovanillone depletion (Supplementary Figure 3). The results on the depletion of monoaromatics from $\mathrm{BL}$ extracts were replicated using the mixture of these compounds in defined medium. Finally, the total amount of compounds consumed correlated with the biomass yield of each strain growing in the mixture (Supplementary Figure 2). Thus, GD01 and GD02, the only two strains that depleted all six compounds, yielded the most biomass on the mixture. Because GD02 grew faster and more reproducibly in flasks than did GD01, it was selected for further studies.

\section{Identification of Pathways Responsible for the Catabolism of BL Aromatic Compounds}

To identify the pathways potentially responsible for the catabolism of monoaromatic compounds in $\mathrm{BL}$, we searched the draft genome sequences of GD01 and GD02 for aromatic catabolic genes using the RHA1 and the EP4 genomes as references. Although the EP4 genome is more similar to that of GD02, many of the aromatic catabolic genes have been validated in RHA1. Several gene clusters and individual genes were identified that encode catabolic pathways for the compounds degraded by GD02 (Figure 1). These include genes encoding guaiacol $O$-demethylase $(g \operatorname{co} A B)$, vanillin dehydrogenase $(v d h)$, vanillate $O$-demethylase $(\operatorname{van} A B)$, and a convergent $\beta$-ketoadipate pathway for the catabolism of catechol and protocatechuate to central metabolites (Supplementary Table 3). Consistent with these predictions, GD02 grew on each of $2 \mathrm{mM}$ vanillate, guaiacol, and vanillin (Supplementary Figure 4), with approximate doubling times of $3.3 \mathrm{~h}$ for the first two substrates and $16 \mathrm{~h}$ for vanillin at $30^{\circ} \mathrm{C}$. As with acetovanillone, growth on vanillin was preceded by a prolonged lag phase.

A striking difference between the GD02 and EP4 genomes despite their high ANI was the absence of $a g c$ and $a p h$ genes in GD02 (Figure 1). These genes are responsible for the catabolism of 4-alkylguaiacols and 4-alkylphenols in EP4 (LevyBooth et al., 2019; Fetherolf et al., 2020). Consistent with this finding, GD02 did not grow on either 4-propylguaiacol or 4-ethylphenol. Indeed, the GD02 genome does not appear to contain any genes encoding extradiol dioxygenases, suggesting that most, if not all, of the aromatic compounds catabolized by this bacterium are funneled through the $\beta$-ketoadipate pathway, which contains intradiol dioxygenases.

To date, there have been no reports describing the catabolism of acetovanillone. However, the catabolism of the chemically related compound, 4-hydroxyacetophenone, has been described in Aromatoleum aromaticum strain EbN1 (Wohlbrand et al., 2008). This catabolism is initiated by a three-component, biotindependent carboxylase, XccBCA, comprising a biotin carboxyl carrier protein (BCCP), a biotin carboxylase (BC), and a carboxyl transferase (CT), respectively. Interestingly, the GD01 and GD02 draft genomes contain genes encoding a homolog of this enzyme, whose subunits share $45-50 \%$ amino acid sequence identity with the EbN1 homologs (Table 2). These genes do not occur in EP4 (Figure 1) which does not grow on acetovanillone (Figure 2A). Based on these observations, we propose that the genes, annotated as apkCBA, encode a carboxylase that is involved in the catabolism of acetovanillone, an alkyl-phenyl ketone, in GD02 and, by extension, GD01. To further understand the catabolism of the monoaromatic compounds by GD02, we monitored their consumption during growth on a mixture of the compounds. Consistent with the growth of GD02 on the individual compounds, guaiacol and vanillate were the first compounds to be consumed (Figure 5). Similarly, vanillin and acetovanillone were consumed after a significant lag phase. 4EG and 4PG were depleted at about the same time as the vanillin was consumed. Interestingly, dihydroferulate was detected in the culture supernatant at this time, suggesting that it was produced by the enzymatic oxidation of $4 \mathrm{PG}$. By contrast, the presumed oxidation product of $4 \mathrm{EG}$, homovanillate, was not detected. Importantly, GD02 did not grow on either 4EG or 4PG. To investigate the ability of GD02 to transform $4 \mathrm{PG}$ and $4 \mathrm{EG}$, cells grown on the mixture of monoaromatics were incubated with each of the alkylguaiacols. As summarized in Supplementary Figure 5, the cells completely transformed 4PG into two compounds, one of each was dihydroferulate, but only partially transformed $4 \mathrm{EG}$ to homovanillate. 
TABLE 2 | Annotation of GD02 gene targets.

\begin{tabular}{|c|c|c|c|c|c|c|}
\hline Gene & Description & Gene ID ${ }^{a}$ & Ref strain & Accession no ${ }^{b}$ & $\% I^{c}$ & References \\
\hline catA & Catechol 1,2-dioxygenase & 3_1052 & $\mathrm{RHA1}$ & WP_009475036.1 & $69.0 \%$ & $\begin{array}{l}\text { Patrauchan et al., } \\
2005\end{array}$ \\
\hline pcaH & $\begin{array}{l}\text { Protocatechuate } \\
\text { 3,4-dioxygenase, } \beta \text { subunit }\end{array}$ & 1_410 & $\mathrm{RHA1}$ & WP_009474041.1 & $48.7 \%$ & $\begin{array}{l}\text { Patrauchan et al., } \\
2005\end{array}$ \\
\hline $\operatorname{van} A^{d}$ & $\begin{array}{l}\text { Vanillate O-demethylase, } \\
\text { oxygenase }\end{array}$ & 3_1042 & $\mathrm{RHA1}$ & WP_011596645.1 & $30.1 \%$ & Chen et al., 2012 \\
\hline$v d h$ & Vanillin dehydrogenase & 2_1324 & $\mathrm{RHA1}$ & WP_011595659.1 & $52.4 \%$ & Chen et al., 2012 \\
\hline$g c O A$ & $\begin{array}{l}\text { Guaiacol O-demethylase, } \\
\text { cytochrome P450 }\end{array}$ & 3_1059 & $\mathrm{RHA1}$ & WP_011595125.1 & $76.4 \%$ & Fetherolf et al., 2020 \\
\hline$a p k C$ & $\begin{array}{l}\text { Putative alkyl-phenyl ketone } \\
\text { carboxylase, } \gamma \text { subunit }\end{array}$ & $13 \_4$ & $\begin{array}{l}\text { Aromatoleum } \\
\text { aromaticum EbN1 }\end{array}$ & CAI06288.1 & $46.5 \%$ & Wohlbrand et al., 2008 \\
\hline apkB & $\begin{array}{l}\text { Putative alkyl-phenyl ketone } \\
\text { carboxylase, } \beta \text { subunit }\end{array}$ & 13_5 & $\begin{array}{l}\text { Aromatoleum } \\
\text { aromaticum EbN1 }\end{array}$ & CAI06287.1 & $50.2 \%$ & Wohlbrand et al., 2008 \\
\hline$a p k A$ & $\begin{array}{l}\text { Putative alkyl-phenyl ketone } \\
\text { carboxylase, } \alpha \text { subunit }\end{array}$ & 13_6 & $\begin{array}{l}\text { Aromatoleum } \\
\text { aromaticum EbN1 }\end{array}$ & CAI06286.1 & $44.8 \%$ & Wohlbrand et al., 2008 \\
\hline
\end{tabular}

${ }^{a}$ Gene ID in GD02 assembly.

'Indicated genes were reciprocal best hits in the reference strain.

'Amino acid sequence identity. In addition, CatA, PcaH, VanA, Vdh, and GcoA were 100\% identical in amino acid sequence to the corresponding gene products in EP4 (Levy-Booth et al., 2019).

${ }^{d}$ The coding sequence in the assembly is $42 \%$ complete. Importantly, vanB is immediately downstream of vanA.

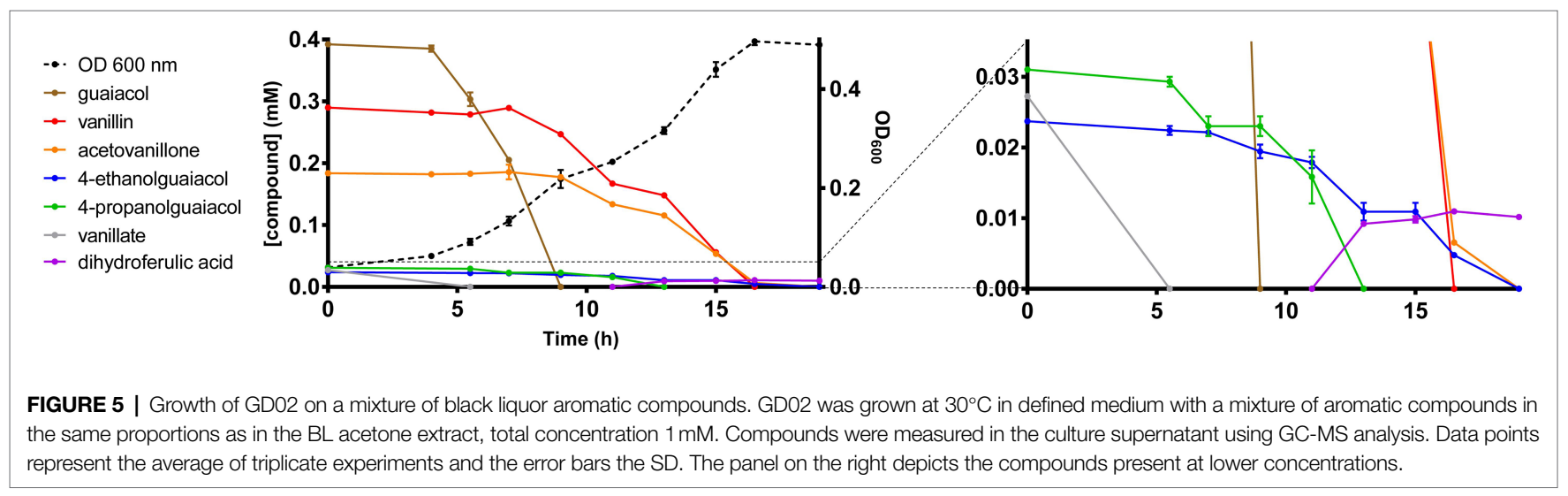

We next evaluated the functionality of the predicted GD02 pathways by using RT-qPCR to assess the expression of select genes (Figure 6A; Table 2) during growth of the bacterium on BL extract. Based on the profile of compound consumption (Figure 5), we sampled RNA of cultures of GD02 growing on a mixture of the BL aromatics at 7 and $11 \mathrm{~h}$. Gene expression was compared to that in GD02 growing on $1 \mathrm{mM}$ citrate. As shown in Figure 6B, vanillin $(v d h)$, guaiacol $(g c o A)$, vanillate $(\operatorname{van} A)$, and acetovanillone $(a p k C)$ catabolic genes were upregulated at least 30 -fold during growth on a mixture of BL monoaromatics vs. citrate. Consistent with the substrate depletion profile (Figure 5), gcoA transcripts were $\sim 100$-fold more abundant at 7 vs. $11 \mathrm{~h}$, while $v d h$ and $a p k C$ transcripts were more abundant at $11 \mathrm{~h}$. Finally, the two tested lower pathway genes were also upregulated during growth on $\mathrm{BL}$ extract. Like gcoA, catA transcripts were more abundant at the earlier sampling time, consistent with the prediction that guaiacol is the only compound catabolized via catechol. The expression profile of $p c a H$ is consistent with the catabolism of vanillin and acetovanillone via the protocatechuate branch of the ortho-cleavage pathway.

\section{DISCUSSION}

Guaiacol, vanillin, and acetovanillone are the most abundant monoaromatic compounds in Lignoforce ${ }^{\mathrm{TM}}$ softwood BL. These are the same major monoaromatic compounds that have been reported in other depolymerized softwood lignin samples, including an oxidative depolymerized Lignoboost softwood kraft lignin (Abdelaziz et al., 2019), an alkaline depolymerized pine kraft lignin (Indulin AT; Prothmann et al., 2017; Ravi et al., 2019), and OCF pine lignin (Zhu et al., 2020). This suggests that these compounds are characteristic monoaromatic compounds generated by oxidative depolymerization of softwood lignin. 4PG and 4EG constituted a relatively minor proportion of the BL monoaromatic compounds reported here ( $\sim 5 \%$ total), but have not been reported in similar softwood lignin samples. This may reflect differences in the fractionation methods or the origin of the lignin. Interestingly, $4 \mathrm{PG}$ is one of the major components of RCF of Eucalyptus (Chen et al., 2020) and corn stover (Fetherolf et al., 2020).

The recovery of monoaromatics and small organic acids, as well as the toxicity of the BL extracts depended on the 
solvent used for the extraction. Thus, increasing the polarity of the solvents, as reflected by the Hansen solubility parameters (Table 1), improved the yield of monoaromatics and small organic acids while at the same time diminishing the toxicity of the extracts. The improved recovery of the monoaromatics and acids using higher polarity solvent is consistent with previous reports (Jipa et al., 2009; Yang et al., 2021). However, there are no studies referencing the toxicity of the extracted compounds to bacteria. The strains examined in this study fall into two phyla, Proteobacteria and Actinobacteria, and have been previously characterized for their abilities to metabolize LDACs (Kosa and Ragauskas, 2012; Salvachúa et al., 2015; Kamimura et al., 2017; Levy-Booth et al., 2019; Ravi et al., 2019). Acetone extracts contained the highest amount of monoaromatics and were the least toxic to bacteria, suggesting that the inhibition of growth was not due to the monoaromatics. The two Proteobacteria, KT2442 and SYK-6, stood out as the only strains not inhibited by any of the four extracts. Interestingly, depolymerized softwood LignoBoost lignin inhibited the growth of R. opacus DSM 1069 but not of KT2440 and SYK-6 (Abdelaziz et al., 2019), in agreement with this study.

The ability of the different bacterial strains to grow on the BL extract, replicated using a mixture of these compounds, is largely consistent with the known or predicted catabolic capabilities of the respective bacteria. For example, vanillin and vanillate were consumed by all five strains, in agreement with the presence of $v d h$ and $v a n A B$ genes in their genomes. Similarly, the three Rhodococcus strains depleted guaiacol, consistent with them encoding GcoAB, a cytochrome P450 system responsible for the $\mathrm{O}$-demethylation of guaiacol to catechol (Fetherolf et al., 2020). The inability of KT2442 and SYK-6 to assimilate guaiacol in a mixture of monoaromatics was also observed in previous reports (Abdelaziz et al., 2019; Ravi et al., 2019). GD01 and GD02 were the only strains that consumed all the identified monoaromatics, which is consistent with these strains reaching the highest $\mathrm{OD}_{600}$ on a mixture of the monoaromatic compounds. SYK-6 was also able to deplete acetovanillone from BL; consistent with a previous report that SYK-6 depletes acetovanillone when growing on a mixture of compounds (Abdelaziz et al., 2019). As demonstrated here, that depletion depends on the presence of vanillate. Further studies are needed to determine the extent and mechanism of this depletion. Although 4PG and 4EG were minor components of BL extracts, it is nevertheless interesting that SYK-6 degraded $4 \mathrm{PG}$ but not 4EG. It is possible that the side chain of $4 \mathrm{PG}$ is oxidized to a carboxylate and further metabolized by the same pathway as ferulate (Masai et al., 2002). 4EG, with its shorter side chain, would not be degraded by this pathway. Further studies are required to test this hypothesis.

GD01 and GD02 were the only strains that grew on all the major monoaromatic compounds in the BL, and are the only strains reported to grow on acetovanillone. These findings are consistent with previous reports by Abdelaziz et al. (2019) and Ravi et al. (2019) in which known bacterial strains were able to grow on depolymerized softwood kraft lignin although none depleted all the monoaromatics. The comparative genomic data, bioinformatic data, and the RT-qPCR analysis strongly suggest that acetovanillone catabolism involves the carboxylation of the acetovanillone moiety, similar to 4-hydroxyacetophenone catabolism in EbN1 (Wohlbrand et al., 2008). Thus, GD01 and
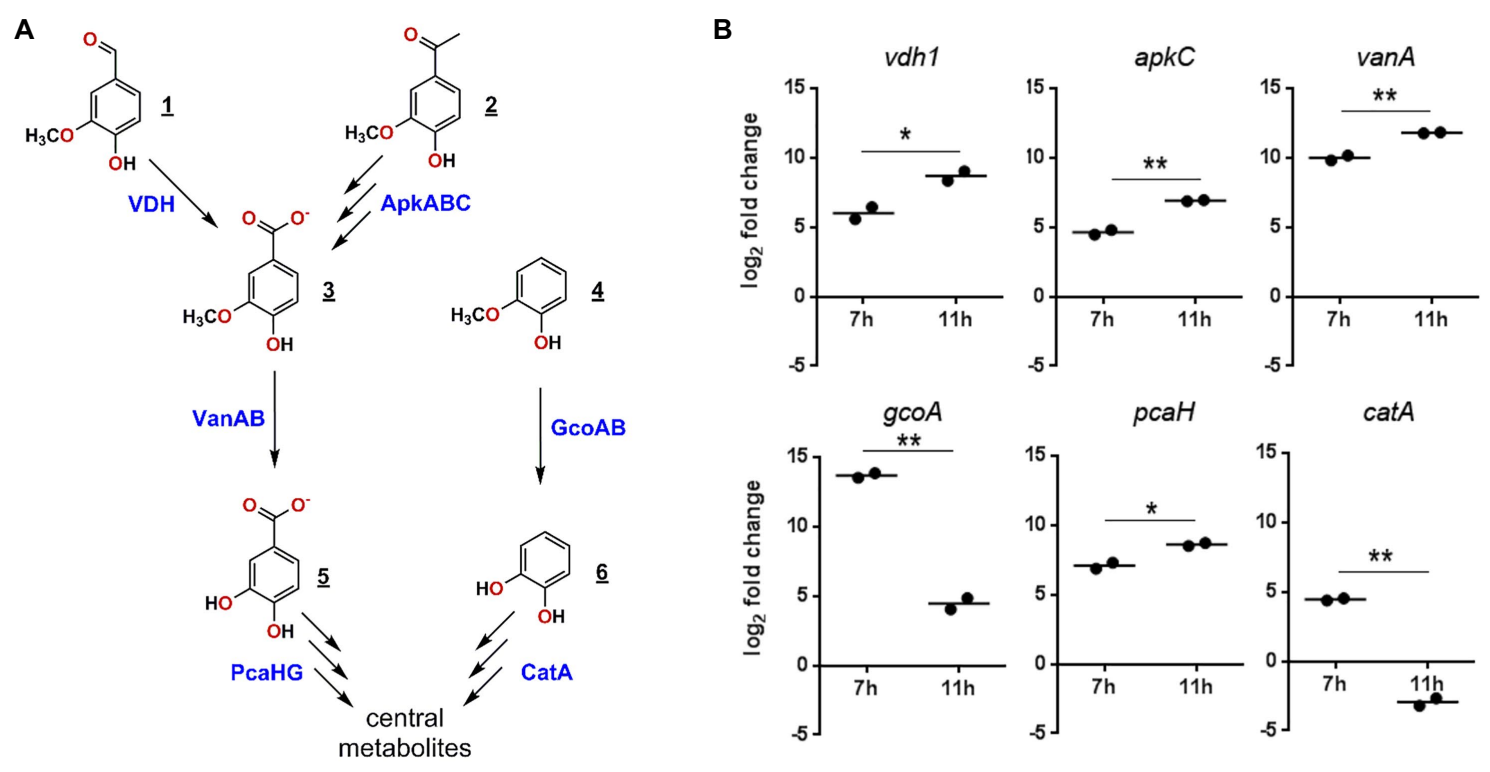

FIGURE 6 | Expression of selected genes in GD02 during growth on a mixture of black liquor compounds. (A) Enzymes predicted to be involved in the catabolism of the major BL monoaromatic compounds and whose genes were investigated using RT-qPCR. Compounds are: 1, vanillin; 2, acetovanillone; 3, vanillate; 4, guaiacol; 5, protocatechuate; and 6, catechol. (B) Expression RT-qPCR of key catabolic genes in GD02 after 7 and $11 \mathrm{~h}$ of growth on $1 \mathrm{mM}$ of a mixture of

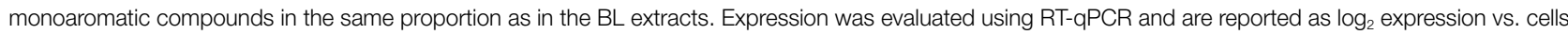
growing on $1 \mathrm{mM}$ citrate after normalization to sigA expression. Values are the average of two technical replicates. Significance was determined by $t$-test, where $p$ values are: ${ }^{*}<0.05,{ }^{* *}<0.01$. 
GD02 consumed acetovanillone, but a very closely related strain, EP4, did not. Notably, the apk genes occur in GD01 and GD02, but not EP4. Consistent with the involvement of the carboxylase in acetovanillone catabolism, $a p k C$ was upregulated during growth on BL extract. While it is unclear how acetovanillone carboxylation contributes to its catabolism, we note that this reaction would yield a $\beta$-keto acid similar to that generated in the catabolism of ferulate by RHA1, which yields vanillate and acetyl-CoA (Otani et al., 2014). Efforts to elucidate acetovanillone catabolism in GD01 and GD02 are ongoing.

The genomic and RT-qPCR analyses further indicate that in GD02, all the major BL LDACs are funneled through the $\beta$-ketoadipate pathway (Figure 6). More specifically, guaiacol is catabolized via the catechol branch of the pathway while vanillin and potentially acetovanillone are catabolized by the protocatechuate branch. Importantly, the $g \operatorname{co} A$ and $v a n A$ transcripts were most highly abundant at the earlier sampling time, consistent with guaiacol and vanillate being the first compounds in the mixture to be depleted. Similarly, the higher abundance of $v d h$ and $a p k C$ during the second sampling time is consistent with the delayed catabolism of vanillin and acetovanillone. The upregulation of vanA and $p c a H$ at the later sampling time is further evidence that vanillin and acetovanillone are catabolized via vanillate and the $\beta$-ketoadipate pathway. Overall, the catabolism of BL LDACs by GD02 provides a striking example of convergence, an organizational principle of the catabolism of aromatic compounds documented in other bacteria, including rhodococci (Eltis and Singh, 2018).

Overall, our results establish that bacteria are able to catabolize the major monoaromatic components of Lignoforce ${ }^{\mathrm{TM}}$ softwood BL. Acetone appears to be the best extraction solvent, recovering the highest quantities of monomers from this liquor stream, and the lowest quantities of growth-inhibiting compounds. Of the five strains that grew on the BL extracts, only GD01 and GD02 catabolized all the identified aromatic monomers. The genes responsible for catabolizing the various LDACs in GD02 were provisionally identified providing a basis for designing biocatalysts to valorize $\mathrm{BL}$ and other under-utilized lignin-rich streams. The ability of GD02 to grow on acetovanillone is of particular interest. More specifically, elucidating the acetovanillone catabolic pathway is critical to exploiting its biocatalytic potential and designing microbial cell factories to valorize lignin streams.

\section{REFERENCES}

Abdelaziz, O. Y., Ravi, K., Mittermeier, F., Meier, S., Riisager, A., Lidén, G., et al. (2019). Oxidative depolymerization of kraft lignin for microbial conversion. ACS Sustain. Chem. Eng. 7, 11640-11652. doi: 10.1021/ acssuschemeng.9b01605

Bankevich, A., Nurk, S., Antipov, D., Gurevich, A. A., Dvorkin, M., Kulikov, A. S., et al. (2012). SPAdes: a new genome assembly algorithm and its applications to single-cell sequencing. J. Comput. Biol. 19, 455-477. doi: 10.1089/cmb.2012.0021

Bauchop, T., and Elsden, S. R. (1960). The growth of micro-organisms in relation to their energy supply. J. Gen. Microbiol. 23, 457-469. doi: 10.1099/00221287-23-3-457

Becker, J., and Wittmann, C. (2019). A field of dreams: lignin valorization into chemicals, materials, fuels, and health-care products. Biotechnol. Adv. 37:107360. doi: 10.1016/j.biotechadv.2019.02.016

\section{DATA AVAILABILITY STATEMENT}

The GD01 and GD02 genome assemblies can be downloaded from NCBI at accessions JAHSQN000000000 and JAHRXG000000000, respectively.

\section{AUTHOR CONTRIBUTIONS}

LN performed genomic and transcriptomic analysis and growth experiments, and co-wrote the manuscript. GD isolated strains GD01 and GD02, performed genome sequencing and annotation experiments, and helped to write the manuscript. DL-B assisted with genome assembly, annotation, and analyses. JL performed the RT-qPCR study. MC and S-KJ prepared and analyzed black liquor extracts. SM and SR designed extraction studies and edited the manuscript. WM helped to design the study and edited the manuscript. LE co-designed the study, helped interpret results, and co-wrote the manuscript. All authors contributed to the article and approved the submitted version.

\section{FUNDING}

This study was supported by a research contract from Genome BC (GEN005) and the BC BioProducts Alliance. LE is the recipient of a Canada Research Chair.

\section{ACKNOWLEDGMENTS}

We thank FPInnovations (Adil Mazar and Michael Paleologou) and Domtar Corporation (Canada) for providing the LignoForce ${ }^{\mathrm{TM}}$ black liquor filtrate. 4PG was kindly provided by Rui Katahira from the National Renewable Energy Laboratory (NREL).

\section{SUPPLEMENTARY MATERIAL}

The Supplementary Material for this article can be found online at: https://www.frontiersin.org/articles/10.3389/fmicb.2021.7350 00/full\#supplementary-material 
Chen, H. P., Chow, M., Liu, C. C., Lau, A., Liu, J., and Eltis, L. D. (2012). Vanillin catabolism in Rhodococcus jostii RHAl. Appl. Environ. Microbiol. 78, 586-588. doi: 10.1128/AEM.06876-11

Chen, X., Zhang, K., Xiao, L. P., Sun, R. C., and Song, G. (2020). Total utilization of lignin and carbohydrates in Eucalyptus grandis: an integrated biorefinery strategy towards phenolics, levulinic acid, and furfural. Biotechnol. Biofuels 13:2. doi: 10.1186/s13068-019-1644-z

Eltis, L. D., and Singh, R. (2018). "Chapter 11. Biological funneling as a means of transforming lignin-derived aromatic compounds into value-added chemicals," in Lignin Valorization: Emerging Approaches. ed. G. T. Beckham (Cambridge: The Royal Society of Chemistry), 290-313.

Fetherolf, M. M., Levy-Booth, D. J., Navas, L. E., Liu, J., Grigg, J. C., Wilson, A., et al. (2020). Characterization of alkylguaiacol-degrading cytochromes P450 for the biocatalytic valorization of lignin. Proc. Natl. Acad. Sci. 117:25771. doi: $10.1073 /$ pnas.1916349117

Hansen, C. M. (2007). Hansen Solubility Parameters: A User's Handbook. 2nd Edn. CRC Press, 1-544.

Hughes, J., Armitage, Y. C., and Symes, K. C. (1998). Application of whole cell rhodococcal biocatalysts in acrylic polymer manufacture. Antonie Van Leeuwenhoek 74, 107-118. doi: 10.1023/A:1001716332272

Jain, C., Rodriguez, R. L., Phillippy, A. M., Konstantinidis, K. T., and Aluru, S. (2018). High throughput ANI analysis of $90 \mathrm{~K}$ prokaryotic genomes reveals clear species boundaries. Nat. Commun. 9:5114. doi: 10.1038/s41467-018-07641-9

Jipa, I., Dobre, T., Stroescu, M., and Stoica, A. (2009). Acetic acid extraction from fermentation broth experimental and modelling studies. Rev. Chim. 60, 1084-1089.

Johnson, C. W., Salvachua, D., Rorrer, N. A., Black, B. A., Vardon, D. R., St. John, P. C., et al. (2019). Innovative chemicals and materials from bacterial aromatic catabolic pathways. Joule 3, 1523-1537. doi: 10.1016/j.joule.2019.05.011

Kamimura, N., Takahashi, K., Mori, K., Araki, T., Fujita, M., Higuchi, Y., et al. (2017). Bacterial catabolism of lignin-derived aromatics: new findings in a recent decade: update on bacterial lignin catabolism. Environ. Microbiol. Rep. 9, 679-705. doi: 10.1111/1758-2229.12597

Kosa, M., and Ragauskas, A. J. (2012). Bioconversion of lignin model compounds with oleaginous Rhodococci. Appl. Microbiol. Biotechnol. 93, 891-900. doi: 10.1007/s00253-011-3743-z

Kouisni, L., Gagné, A., Maki, K., Holt-Hindle, P., and Paleologou, M. (2016). LignoForce system for the recovery of lignin from black liquor: feedstock options, odor profile, and product characterization. ACS Sustain. Chem. Eng. 4, 5152-5159. doi: 10.1021/acssuschemeng.6b00907

Krell, T., Lacal, J., Guazzaroni, M. E., Busch, A., Silva-Jimenez, H., Fillet, S., et al. (2012). Responses of pseudomonas putida to toxic aromatic carbon sources. J. Biotechnol. 160, 25-32. doi: 10.1016/j.jbiotec.2012.01.026

Lane, D. J. (1991). "16S/23S rRNA sequencing” in Nucleic Acid Techniques in Bacterial Systematics. eds. E. Stackebrandt and M. Goodfellow (New York, NY: John Wiley \& Sons, Inc.), 115-175.

Levy-Booth, D. J., Fetherolf, M. M., Stewart, G. R., Liu, J., Eltis, L. D., and Mohn, W. W. (2019). Catabolism of Alkylphenols in Rhodococcus via a meta-cleavage pathway associated with Genomic Islands. Front. Microbiol. 10:1862. doi: $10.3389 /$ fmicb.2019.01862

Masai, E., Harada, K., Peng, X., Kitayama, H., Katayama, Y., and Fukuda, M. (2002). Cloning and characterization of the ferulic acid catabolic genes of Sphingomonas paucimobilis SYK-6. Appl. Environ. Microbiol. 68, 4416-4424. doi: 10.1128/AEM.68.9.4416-4424.2002

Mathews, S. L., Grunden, A. M., and Pawlak, J. (2016). Degradation of lignocellulose and lignin by Paenibacillus glucanolyticus. Int. Biodeterior. Biodegradation 110, 79-86. doi: 10.1016/j.ibiod.2016.02.012

Mathews, S. L., Pawlak, J. J., and Grunden, A. M. (2014). Isolation of Paenibacillus glucanolyticus from pulp mill sources with potential to deconstruct pulping waste. Bioresour. Technol. 164, 100-105. doi: 10.1016/j.biortech.2014.04.093

Otani, H., Lee, Y. E., Casabon, I., and Eltis, L. D. (2014). Characterization of p-hydroxycinnamate catabolism in a soil Actinobacterium. J. Bacteriol. 196, 4293-4303. doi: 10.1128/JB.02247-14

Patrauchan, M. A., Florizone, C., Dosanjh, M., Mohn, W. W., Davies, J., and Eltis, L. D. (2005). Catabolism of benzoate and phthalate in Rhodococcus sp. strain RHA1: redundancies and convergence. J. Bacteriol. 187, 4050-4063. doi: 10.1128/JB.187.12.4050-4063.2005

Pepper, J. M., Sundaram, G. S., and Dyson, G. (1971). Lignin and related compounds. III. An improved synthesis of 3-(4-hydroxy-3-methoxyphenyl)-1propanol and 3-(4-hydroxy-3,5-dimethoxyphenyl)-1-propanol. Can. J. Chem. 49, 3394-3395. doi: 10.1139/v71-564
Perez, J. M., Kontur, W. S., Gehl, C., Gille, D. M., Ma, Y., Niles, A. V., et al. (2021). Redundancy in aromatic O-demethylation and ring opening reactions in Novosphingobium aromaticivorans and their impact in the metabolism of plant derived phenolics. Appl. Environ. Microbiol. 87, e02794-e02720. doi: 10.1128/AEM.02794-20

Prothmann, J., Sun, M., Spegel, P., Sandahl, M., and Turner, C. (2017). Ultrahigh-performance supercritical fluid chromatography with quadrupole-timeof-flight mass spectrometry (UHPSFC/QTOF-MS) for analysis of lignin-derived monomeric compounds in processed lignin samples. Anal. Bioanal. Chem. 409, 7049-7061. doi: 10.1007/s00216-017-0663-5

Ragauskas, A. J., Beckham, G. T., Biddy, M. J., Chandra, R., Chen, F., Davis, M. F., et al. (2014). Lignin valorization: improving lignin processing in the biorefinery. Science 344:1246843. doi: 10.1126/science.1246843

Ravi, K., Abdelaziz, O. Y., Nobel, M., Garcia-Hidalgo, J., Gorwa-Grauslund, M. F., Hulteberg, C. P., et al. (2019). Bacterial conversion of depolymerized kraft lignin. Biotechnol. Biofuels 12:56. doi: 10.1186/s13068-019-1397-8

Salvachúa, D., Karp, E. M., Nimlos, C. T., Vardon, D. R., and Beckham, G. T. (2015). Towards lignin consolidated bioprocessing: simultaneous lignin depolymerization and product generation by bacteria. Green Chem. 17, 4951-4967. doi: 10.1039/C5GC01165E

Schutyser, W., Renders, T., Van den Bosch, S., Koelewijn, S.-F., Beckham, G. T., and Sels, B. F. (2018). Chemicals from lignin: an interplay of lignocellulose fractionation, depolymerisation, and upgrading. Chem. Soc. Rev. 47, 852-908. doi: 10.1039/C7CS00566K

Sun, Z., Fridrich, B., de Santi, A., Elangovan, S., and Barta, K. (2018). Bright side of lignin depolymerization: toward new platform chemicals. Chem. Soc. Rev. 118, 614-678. doi: 10.1021/acs.chemrev.7b00588

Suzuki, Y., Otsuka, Y., Araki, T., Kamimura, N., Masai, E., Nakamura, M., et al. (2021). Lignin valorization through efficient microbial production of beta-ketoadipate from industrial black liquor. Bioresour. Technol. 337:125489. doi: 10.1016/j.biortech.2021.125489

Vardon, D. R., Franden, M. A., Johnson, C. W., Karp, E. M., Guarnieri, M. T., Linger, J. G., et al. (2015). Adipic acid production from lignin. Energy Environ. Sci. 8, 617-628. doi: 10.1039/C4EE03230F

Weimer, A., Kohlstedt, M., Volke, D. C., Nikel, P. I., and Wittmann, C. (2020). Industrial biotechnology of Pseudomonas putida: advances and prospects. Appl. Microbiol. Biotechnol. 104, 7745-7766. doi: 10.1007/s00253-020-10811-9

Wohlbrand, L., Wilkes, H., Halder, T., and Rabus, R. (2008). Anaerobic degradation of p-ethylphenol by "Aromatoleum aromaticum" strain EbN1: pathway, regulation, and involved proteins. J. Bacteriol. 190, 5699-5709. doi: 10.1128/ JB.00409-08

Xu, Z., Lei, P., Zhai, R., Wen, Z., and Jin, M. (2019). Recent advances in lignin valorization with bacterial cultures: microorganisms, metabolic pathways, and bio-products. Biotechnol. Biofuels 12:32. doi: 10.1186/s13068-019-1376-0

Yang, W., Wang, X., Ni, S., Liu, X., Liu, R., Hu, C., et al. (2021). Effective extraction of aromatic monomers from lignin oil using a binary petroleum ether/dichloromethane solvent. Sep. Purif. Technol. 267:118599. doi: 10.1016/j. seppur.2021.118599

Zhu, Y., Liao, Y., Lv, W., Liu, J., Song, X., Chen, L., et al. (2020). Complementing vanillin and cellulose production by oxidation of lignocellulose with stirring control. ACS Sustain. Chem. Eng. 8, 2361-2374. doi: 10.1021/acssuschemeng.9b04837

Conflict of Interest: The authors declare that the research was conducted in the absence of any commercial or financial relationships that could be construed as a potential conflict of interest.

Publisher's Note: All claims expressed in this article are solely those of the authors and do not necessarily represent those of their affiliated organizations, or those of the publisher, the editors and the reviewers. Any product that may be evaluated in this article, or claim that may be made by its manufacturer, is not guaranteed or endorsed by the publisher.

Copyright (C) 2021 Navas, Dexter, Liu, Levy-Booth, Cho, Jang, Mansfield, Renneckar, Mohn and Eltis. This is an open-access article distributed under the terms of the Creative Commons Attribution License (CC BY). The use, distribution or reproduction in other forums is permitted, provided the original author(s) and the copyright owner(s) are credited and that the original publication in this journal is cited, in accordance with accepted academic practice. No use, distribution or reproduction is permitted which does not comply with these terms. 